\title{
Estimation of CE-CVM energy parameters from miscibility gap data
}

\author{
G SRINIVASA GUPTA, G VAMSI MADHAV, A PANDEY, B NAGESWARA SARMA* \\ and S LELE
}

Centre of Advanced Study, Department of Metallurgical Engineering, Institute of Technology, Banaras Hindu University, Varanasi 221005 , India

MS received 10 May 2004

\begin{abstract}
The powerful framework of cluster expansion-cluster variation methods (CE-CVM) expresses alloy free energy in terms of energy (model) parameters, macroscopic variables (composition and temperature) and microscopic variables (correlation functions). A simultaneous optimization of thermodynamic and phase equilibria data using CE-CVM is critically dependent on giving good initial values of energy parameters, macroscopic and microscopic variables, respectively. No standard method for obtaining the initial values of the energy parameters is available in literature. As a starting point, a method has been devised to estimate the values of energy parameters from consolute point (miscibility gap maximum) data. Empirical relations among energy parameters, temperature $\left(T_{c}\right)$, composition $\left(x_{c}\right)$ and $d^{2} T / d x^{2}$ at the consolute point, have been developed using CE-CVM free energy functions for $b c c$ and $f c c$ structures in the tetrahedron and tetrahedronoctahedron approximations, respectively. Thus from the observed data of $T_{c}, x_{c}$ and $d^{2} T / d x^{2}$ in the above relations, good initial values of energy parameters can be obtained. Further, a necessary modification to the classical NR method for solving simultaneous nonlinear/transcendental equations with a double root in one variable and a simple root in the other has been presented.
\end{abstract}

Keywords. Cluster expansion method; cluster variation method; bcc and fcc structures; consolute point; miscibility gap; Newton-Raphson method.

\section{Introduction}

The framework of cluster expansion (CE) method due to Sanchez et al (1984) and cluster variation method (CVM) proposed by Kikuchi (1951) offers a physically meaningful and mathematically tractable hierarchy of approximations to handle the ever-present short range order in materials systems. It also subsumes many of the classical approximations of the alloy problem as lower levels of the hierarchy. In fact, CE-CVM has become the method of choice for computation of phase diagrams and thermodynamics of materials systems using the energy parameters estimated from first-principles quantum mechanical calculations (de Fontaine 1994). While the first-principles calculations have been quite successful in obtaining the correct topologies of the phase diagrams, the quantitative agreement with the observed data needs significant improvement (Saunders and Miodownik 1998). In view of this, it becomes important to be able to find an optimized set of model parameters for the case of standard CECVM approximations in different materials systems. These parameters will characterize the chosen alloy system if they are found by simultaneous (nonlinear) optimization of all types of available thermodynamic and phase equi-

*Author for correspondence (drbnsarma@yahoo.com) libria data. Success of these nonlinear optimization procedures critically depends on the ability to provide good initial values for the model parameters. If appropriate initial values are not chosen, the topology of the phase diagram will be different from that of the observed one. Under such circumstances, the errors (the deviations between the calculated and observed compositions/temperatures) cannot even be evaluated, leading to a breakdown of the optimization procedure. Even so, there is no standard procedure for estimating the initial values of CE-CVM model parameters available in literature. In this communication, we present the details of an empirical procedure using which the model parameters can be estimated from the miscibility gap data in binary systems. In $\S 2$, a brief outline of the CE-CVM formulations of free energy for disordered binary $b c c$ and $f c c$ structures is presented. A modified Newton-Raphson method for the determination of consolute point (miscibility gap maximum) is presented in $\S 3$, whereas an empirical procedure for the estimation of CE-CVM energy parameters from miscibility gap data is given in $\S 4$.

\section{CE-CVM formulations for $b c c$ and $f c c$ structures}

For the sake of completeness, a brief outline of the CECVM formulations for disordered binary bcc (Ackerman 
et al 1989) and fcc (Sanchez and de Fontaine 1978) structures, respectively under tetrahedron and tetrahedronoctahedron approximations is given below. An excellent review on this subject by Inden and Pitsch (1991) may be consulted for the details of cluster algebra. As usual, we shall present the results for a typical choice of energy parameters in an orthogonal basis corresponding to the following relations among the site operator, $\sigma_{i}$, and the site occupation operators $p_{i}^{A}$ and $p_{i}^{B}$ :

$$
\left[\begin{array}{c}
1 \\
\sigma_{i}
\end{array}\right]=\left[\begin{array}{rr}
1 & 1 \\
-1 & 1
\end{array}\right]\left[\begin{array}{l}
p_{i}^{A} \\
p_{i}^{B}
\end{array}\right] .
$$

The four vertices of the tetrahedron motif in $b c c$ are labelled 1, 2, 3 and 4, respectively such that 12, 23, 34 and 14 distances represent first neighbour pairs while 13 and 24 represent second neighbour pairs. All the triangles forming part of the motif are crystallographically identical. Accordingly we have the following correlation functions $\left(u_{i}\right):\left\langle\sigma_{1} \sigma_{2}\right\rangle,\left\langle\sigma_{1} \sigma_{3}\right\rangle,\left\langle\sigma_{1} \sigma_{2} \sigma_{3}\right\rangle,\left\langle\sigma_{1} \sigma_{2} \sigma_{3} \sigma_{4}\right\rangle$ and $\left\langle\sigma_{1}\right\rangle$. Of these, the point correlation function $\left\langle\sigma_{1}\right\rangle$ is related to composition through the relation $\left\langle\sigma_{1}\right\rangle=-x_{A}+x_{B}=2 x_{B}-1$ and is fixed for an alloy of fixed composition. Thus, we have 4 independent correlation functions which describe the microscopic state of the system. The probabilities of occurrence of different types of atomic arrangements on the motif/submotif sites are called cluster variables. There are a total of 20 distinct clusters present in the structure. These cluster probabilities are linear functions of $u_{i}$.

Following the $\mathrm{CE}$ method, the configurational energy of the system is expressed as a bilinear sum of the products of the $u_{i}$ (along with the appropriate multiplicities of the submotifs) and the corresponding energy coefficients, $e_{i}$. The configurational entropy can be obtained by using CVM through appropriate linear combinations of the Boltzmann entropy summations of cluster probabilities corresponding to each of the motif/submotifs, such that each of the cluster/subcluster configurations is counted only once. This is done through the so-called KikuchiBarker overlap correction coefficients (Kikuchi 1951; Barker 1953).

Thus the free energy of the system is expressed as a function of the model energy parameters $\left(e_{i}\right)$, the macroscopic variables (composition, $x_{B}$ and temperature, $T$ ) and the microscopic internal variables $\left(u_{i}\right)$ in a hierarchical manner. Similar formulation has been carried out for disordered binary $f c c$ structure using regular tetrahedron and octahedron as motifs (Sanchez and de Fontaine 1978). In this case, there are a total of 9 correlation functions (and hence 9 energy parameters) apart from the point correlation function. These correspond to first neighbour pair, second neighbour pair, basal (equilateral) triangle, lateral (isosceles) triangle, regular tetrahedron, irregular tetrahedron, square, 5-point pyramid and octahedron. The free energy expression is obtained as in the case of $b c c$.

\section{Determination of consolute point}

The composition, $x_{\mathrm{c}}$ and temperature, $T_{\mathrm{c}}$, of the consolute point can be determined from the following set of two equations (Lupis 1983):

$$
\frac{\partial^{2} G}{\partial x^{2}}=0
$$

and

$$
\frac{\partial^{3} G}{\partial x^{3}}=0
$$

where $G$ is a function of $x$ and $T$. Introducing standard notation for partial derivatives, these equations can be written as

$$
G_{x x}=0
$$

and

$$
G_{x x x}=0 .
$$

Explicit solutions for $x_{\mathrm{c}}$ and $T_{\mathrm{c}}$ as given, for example, by Lupis (1983) cannot be found in the present case, because the internal variables $u_{i}$ have an implicit variation with $x$ and $T$. Hence, they have to be determined for each $x$ and $T$ by minimizing $G$. Thus, $x_{\mathrm{c}}$ and $T_{\mathrm{c}}$ can be found by using numerical procedures such as the NewtonRaphson (NR) method by a simultaneous solution of equations in (2) and (3). Since the second and third partial derivatives of $G$ have to be simultaneously zero at the consolute point, we have a double root in $x$ but a simple root in $T$ (Mathews 1998). The NR procedure needs to be modified for such cases, as given below.

Consider a function, $f(x, T)$, which has a double root at $x_{0}$ in $x$ and a simple root at $T_{0}$ in $T$. Further, consider a Taylor expansion of $f(x, T)$ and $f_{x}(x, T)$ around $x$ and $T$.

$$
\begin{aligned}
& f\left(x_{0}, T_{0}\right)=f(x, T)+\left(x_{0}-x\right) f_{x}(x, T)+\frac{1}{2}\left(x_{0}-x\right)^{2} \\
& f_{x x}(x, T)+\left(x_{0}-x\right)\left(T_{0}-T\right) f_{x T}(x, T)+\left(T_{0}-T\right) f_{T}(x, T),
\end{aligned}
$$

$$
f_{x}\left(x_{0}, T_{0}\right)=f_{x}(x, T)+\left(x_{0}-x\right) f_{x x}(x, T)+\left(T_{0}-T\right) f_{x T}(x, T) .
$$

Note that only the first order deviations in $x$ and $T$ are considered in (5). All the derivatives present in (5) and lower ones are retained in (4). Solving (5) for $\left(T_{0}-T\right)$ after setting the left hand side to zero, we have

$$
\left(T_{0}-T\right)=-\frac{f_{x}+\left(x_{0}-x\right) f_{x x}}{f_{x T}} .
$$

Substituting (6) in (4) and simplifying, we obtain

$$
f_{x x} f_{x T}\left(x_{0}-x\right)^{2}+2 f_{x x} f_{T}\left(x_{0}-x\right)-2\left(f f_{x T}-f_{x} f_{T}\right)=0 .
$$


This is a quadratic in $\left(x_{0}-x\right)$ and can be easily solved to yield the Newton step for $x$.

$$
\left(x_{0}-x\right)=\frac{-f_{x x} f_{T} \pm \sqrt{\left(f_{x x} f_{T}\right)^{2}+2 f_{x x} f_{x T}\left(f f_{x T}-f_{x} f_{T}\right)}}{f_{x x} f_{x T}} .
$$

For positive (negative) values of $f_{x x} f_{T}$, the positive (negative) sign should be selected for the discriminant. Substituting (8) in (6) then yields an expression for $\left(T_{0}-\right.$ $T)$. For very small departures from the correct solution, $f_{x x} f_{T}$ can be taken out as a common factor from the discriminant in (8), the remainder can be expanded using binomial theorem and retaining only the leading term in the same to obtain

$$
\left(x_{0}-x\right)=\left(f f_{x T}-f_{x} f_{T}\right) /\left(f_{x x} f_{T}\right) .
$$

Note that the above method can be utilized to find the consolute point in which $f(x, T)=G_{x x}$ and $f_{x}(x, T)=G_{x x x}$. The NR iterations are carried out using these corrections till a suitable convergence criterion (e.g. the magnitudes of $G_{x x}$ and $G_{x x x}$ are less than, say, 0.001) is met.

\section{Estimation of energy parameters from miscibility gap data}

Consolute point data consist of the critical temperature, $T_{\mathrm{c}}$, composition, $x_{\mathrm{c}}$ and the curvature of the miscibility gap boundary at the consolute point, which is related to $\left(\mathrm{d}^{2} T / \mathrm{d} x^{2}\right)_{\left(T_{\mathrm{c}}, x_{\mathrm{c}}\right)}=T_{x x}$. This $T_{x x}$ is in fact equal to $(1 / 3)$ $\left[\left(\partial^{4} G / \partial x^{4}\right) /\left(\partial^{2} S / \partial x^{2}\right)\right]$ [see Lupis (1983) for details]. These quantities have been obtained for $b c c$ disordered structure in the tetrahedron approximation by using the modified Newton-Raphson method presented in the previous section for an initial choice of energy parameters, $e_{1}=-250 \mathrm{~J} \mathrm{~mol}^{-1}$, $e_{2}=2 e_{1} / 3$ and $e_{3}=e_{4}=0$. These are $T_{\mathrm{c}}=305.825 \mathrm{~K}, x_{\mathrm{c}}=$ 0.5 and $T_{x x}=-397.209 \mathrm{~K}$. This choice of $e_{2}=2 e_{1} / 3$ makes the total contribution of first and second neighbour pairs to the enthalpy of $b c c$ structure comparable to that from first neighbours in $f c c$ structure. By varying the ratios, $e_{3} / e_{1}$ and $e_{4} / e_{1}$, in the range from 0.2 to -0.2 in steps of $0 \cdot 1$, the consolute point data mentioned above have been obtained and given in table 1 .

By fitting suitable polynomial functions of $e_{3} / e_{1}$ and $e_{4} / e_{1}$ to the tabulated data for $T_{\mathrm{c}}, x_{\mathrm{c}}$ and $T_{x x}$, the following relations have been obtained.

$$
\begin{aligned}
x_{c}^{\mathrm{obs}=}= & 0 \cdot 5+0 \cdot 394714\left(e_{3} / e_{1}\right)^{3 / 5}+0 \cdot 536611\left(e_{3} / e_{1}\right)^{3 / 5}\left(e_{4} / e_{1}\right), \\
T_{c}^{\mathrm{obs}}= & e_{1}\left[-1 \cdot 2233-5 \cdot 25314\left(e_{3} / e_{1}\right)^{2}-0.763127\left(e_{4} / e_{1}\right)\right. \\
& \left.-4 \cdot 46246\left(e_{3} / e_{1}\right)^{2}\left(e_{4} / e_{1}\right)\right],
\end{aligned}
$$

$$
\begin{aligned}
T_{x x}^{\mathrm{obs}}= & e_{1}\left[1 \cdot 58884+30.9369\left(e_{3} / e_{1}\right)^{2}-1.73767\left(e_{4} / e_{1}\right)\right. \\
& \left.+83.8545\left(e_{3} / e_{1}\right)^{2}\left(e_{4} / e_{1}\right)\right] .
\end{aligned}
$$

Division of (11) with (12) eliminates the individual $e_{1}$ term and gives the following equation in $e_{3} / e_{1}$ and $e_{4} / e_{1}$.

$$
\begin{aligned}
& \left(5 \cdot 25314 T_{x x}^{\mathrm{obs}}+30 \cdot 9369 T_{c}^{\mathrm{obs}}\right)\left(e_{3} / e_{1}\right)^{2}+\left(0.763127 T_{x x}^{\mathrm{obs}}\right. \\
& \left.-1.73767 T_{c}^{\mathrm{obs}}\right)\left(e_{4} / e_{1}\right)+\left(4 \cdot 46246 T_{x x}^{\mathrm{obs}}+83.8545 T_{c}^{\mathrm{obs}}\right) \\
& \left(e_{3} / e_{1}\right)^{2}\left(e_{4} / e_{1}\right)+1 \cdot 2233 T_{x x}^{\mathrm{obs}}+1.58884 T_{c}^{\mathrm{obs}}=0 .
\end{aligned}
$$

By substituting $e_{4} / e_{1}=0$ in (10) and solving for $e_{3} / e_{1}$, we get an approximate value for $e_{3} / e_{1}$. These can be used as initial values for simultaneously solving (10) and (13) for $e_{3} / e_{1}$ and $e_{4} / e_{1}$ using two-dimensional Newton-Raphson method. Substitution of these in (11) or (12) gives $e_{1}$, from which $e_{3}$ and $e_{4}$ can be found.

The energy parameters thus found can be used for obtaining the corresponding consolute point data for comparison with the observed data in order to test the validity of this procedure. Results of a couple of such tests are summarized in table 2. Comparison of observed and calculated data reveals that the differences in calculated and observed data are well within tolerable limits and the topology of the phase diagram can be reproduced very

Table 1. Consolute point data for disordered $b c c$ structure in the tetrahedron approximation.

\begin{tabular}{rrrll}
\hline$e_{3} / e_{1}$ & $e_{4} / e_{1}$ & \multicolumn{1}{c}{$T_{\mathrm{c}}(\mathrm{K})$} & \multicolumn{1}{c}{$x_{\mathrm{c}}$} & \multicolumn{1}{c}{$T_{x x}(\mathrm{~K})$} \\
\hline $0 \cdot 2$ & $0 \cdot 2$ & $406 \cdot 179$ & $0 \cdot 685289$ & $-799 \cdot 585$ \\
$0 \cdot 2$ & $0 \cdot 1$ & $380 \cdot 245$ & $0 \cdot 668601$ & $-725 \cdot 385$ \\
$0 \cdot 2$ & $0 \cdot 0$ & $355 \cdot 789$ & $0 \cdot 650946$ & $-675 \cdot 977$ \\
$0 \cdot 2$ & $-0 \cdot 1$ & $333 \cdot 092$ & $0 \cdot 633034$ & $-650 \cdot 112$ \\
$0 \cdot 2$ & $-0 \cdot 2$ & $312 \cdot 543$ & $0 \cdot 615662$ & $-646 \cdot 647$ \\
$0 \cdot 1$ & $0 \cdot 2$ & $364 \cdot 967$ & $0 \cdot 63705$ & $-494 \cdot 972$ \\
$0 \cdot 1$ & $0 \cdot 1$ & $341 \cdot 936$ & $0 \cdot 615201$ & $-478 \cdot 627$ \\
$0 \cdot 1$ & $0 \cdot 0$ & $320 \cdot 37$ & $0 \cdot 595764$ & $-485 \cdot 075$ \\
$0 \cdot 1$ & $-0 \cdot 1$ & $300 \cdot 497$ & $0 \cdot 579158$ & $-508 \cdot 214$ \\
$0 \cdot 1$ & $-0 \cdot 2$ & $282 \cdot 755$ & $0 \cdot 565332$ & $-546 \cdot 61$ \\
$0 \cdot 0$ & $0 \cdot 2$ & $345 \cdot 203$ & $0 \cdot 5$ & $-279 \cdot 681$ \\
$0 \cdot 0$ & $0 \cdot 1$ & $325 \cdot 147$ & $0 \cdot 5$ & $-341 \cdot 866$ \\
$0 \cdot 0$ & $0 \cdot 0$ & $305 \cdot 825$ & $0 \cdot 5$ & $-397 \cdot 209$ \\
$0 \cdot 0$ & $-0 \cdot 1$ & $287 \cdot 715$ & $0 \cdot 5$ & $-451 \cdot 324$ \\
$0 \cdot 0$ & $-0 \cdot 2$ & $271 \cdot 472$ & $0 \cdot 5$ & $-511 \cdot 098$ \\
$-0 \cdot 1$ & $0 \cdot 2$ & 364.967 & $0 \cdot 36295$ & -494.972 \\
$-0 \cdot 1$ & $0 \cdot 1$ & $341 \cdot 936$ & $0 \cdot 384799$ & $-478 \cdot 627$ \\
$-0 \cdot 1$ & $0 \cdot 0$ & $320 \cdot 370$ & $0 \cdot 404236$ & $-485 \cdot 075$ \\
$-0 \cdot 1$ & $-0 \cdot 1$ & $300 \cdot 497$ & $0 \cdot 420842$ & $-508 \cdot 214$ \\
$-0 \cdot 1$ & $-0 \cdot 2$ & $282 \cdot 755$ & $0 \cdot 434668$ & $-546 \cdot 61$ \\
$-0 \cdot 2$ & $0 \cdot 2$ & $406 \cdot 179$ & $0 \cdot 314711$ & $-799 \cdot 585$ \\
$-0 \cdot 2$ & $0 \cdot 1$ & $380 \cdot 245$ & $0 \cdot 331399$ & $-725 \cdot 385$ \\
$-0 \cdot 2$ & $0 \cdot 0$ & $355 \cdot 789$ & $0 \cdot 349054$ & $-675 \cdot 977$ \\
$-0 \cdot 2$ & $-0 \cdot 1$ & $333 \cdot 092$ & $0 \cdot 366966$ & $-650 \cdot 112$ \\
$-0 \cdot 2$ & $-0 \cdot 2$ & $312 \cdot 543$ & $0 \cdot 384338$ & $-646 \cdot 647$ \\
\hline & & & & \\
\hline
\end{tabular}


Table 2. Comparison of observed and calculated data for $b c c$ structure.

\begin{tabular}{lccl}
\hline $\begin{array}{l}\text { Initial choice of energies } \\
\left(\mathrm{J} \mathrm{mol}^{-1}\right)\end{array}$ & $\begin{array}{c}\text { Consolute point data for the } \\
\text { initial choice of energies }\end{array}$ & $\begin{array}{c}\text { Energies calculated from } \\
(10)-(13)\left(\mathrm{J} \mathrm{mol}^{-1}\right)\end{array}$ & $\begin{array}{c}\text { Consolute point data } \\
\text { for the calculated energies }\end{array}$ \\
\hline$e_{1}=-250$ & $T_{\mathrm{c}}=312.543 \mathrm{~K}$ & $e_{1}=-245.998$ & $T_{\mathrm{c}}=312.747 \mathrm{~K}$ \\
$e_{2}=-500 / 3$ & $x_{\mathrm{c}}=0.38433$ & $e_{2}=-163.999$ & $x_{\mathrm{c}}=0.379574$ \\
$e_{3}=50$ & $T_{x x}=-646.64 \mathrm{~K}$ & $e_{3}=49 \cdot 6889$ & $T_{x x}=-637.831 \mathrm{~K}$ \\
$e_{4}=50$ & & $e_{4}=43.4941$ & \\
$e_{1}=-250$ & $T_{\mathrm{c}}=355.89 \mathrm{~K}$ & $e_{1}=-247.511$ & $T_{\mathrm{c}}=354.463 \mathrm{~K}$ \\
$e_{2}=-500 / 3$ & $x_{\mathrm{c}}=0.349054$ & $e_{2}=-165.007$ & $x_{\mathrm{c}}=0.348275$ \\
$e_{3}=50$ & $T_{x x}=-675.57 \mathrm{~K}$ & $e_{3}=46.805$ & $T_{x x}=-656.571 \mathrm{~K}$ \\
$e_{4}=0$ & & $e_{4}=-7.05899$ & \\
\hline
\end{tabular}

Table 3. Consolute point data for disordered $f c c$ structure.

\begin{tabular}{|c|c|c|c|c|}
\hline$e_{3} / e_{1}$ & $e_{5} / e_{1}$ & $T_{\mathrm{c}}(\mathrm{K})$ & $x_{\mathrm{c}}$ & $T_{\mathrm{xx}}(\mathrm{K})$ \\
\hline $0 \cdot 2$ & $0 \cdot 2$ & 327.975 & 0.634889 & -433.94 \\
\hline $0 \cdot 2$ & $0 \cdot 1$ & $325 \cdot 959$ & 0.625987 & $-458 \cdot 815$ \\
\hline $0 \cdot 2$ & 0.0 & 324.083 & 0.617829 & $-489 \cdot 58$ \\
\hline 0.2 & $-0 \cdot 1$ & $322 \cdot 339$ & 0.610353 & $-526 \cdot 321$ \\
\hline $0 \cdot 2$ & $-0 \cdot 2$ & $320 \cdot 729$ & 0.603475 & $-569 \cdot 23$ \\
\hline $0 \cdot 1$ & $0 \cdot 2$ & $310 \cdot 185$ & $0 \cdot 580431$ & $-325 \cdot 856$ \\
\hline $0 \cdot 1$ & $0 \cdot 1$ & $308 \cdot 656$ & $0 \cdot 573463$ & $-359 \cdot 551$ \\
\hline $0 \cdot 1$ & $0 \cdot 0$ & $307 \cdot 17$ & 0.567457 & $-398 \cdot 323$ \\
\hline $0 \cdot 1$ & $-0 \cdot 1$ & $305 \cdot 725$ & $0 \cdot 562235$ & $-442 \cdot 574$ \\
\hline $0 \cdot 1$ & $-0 \cdot 2$ & $304 \cdot 333$ & $0 \cdot 557636$ & $-492 \cdot 867$ \\
\hline $0 \cdot 0$ & $0 \cdot 2$ & $303 \cdot 447$ & $0 \cdot 5$ & $-280 \cdot 81$ \\
\hline $0 \cdot 0$ & $0 \cdot 1$ & $302 \cdot 185$ & $0 \cdot 5$ & $-319 \cdot 389$ \\
\hline $0 \cdot 0$ & $0 \cdot 0$ & $300 \cdot 903$ & $0 \cdot 5$ & $-362 \cdot 215$ \\
\hline $0 \cdot 0$ & $-0 \cdot 1$ & $299 \cdot 611$ & 0.5 & $-410 \cdot 004$ \\
\hline $0 \cdot 0$ & $-0 \cdot 2$ & $298 \cdot 326$ & $0 \cdot 5$ & $-463 \cdot 596$ \\
\hline$-0 \cdot 1$ & $0 \cdot 2$ & $310 \cdot 185$ & 0.419569 & $-325 \cdot 856$ \\
\hline$-0 \cdot 1$ & $0 \cdot 1$ & $308 \cdot 656$ & 0.426537 & -359.551 \\
\hline$-0 \cdot 1$ & $0 \cdot 0$ & $307 \cdot 170$ & 0.432543 & $-398 \cdot 323$ \\
\hline$-0 \cdot 1$ & $-0 \cdot 1$ & $305 \cdot 725$ & 0.437765 & $-442 \cdot 574$ \\
\hline$-0 \cdot 1$ & $-0 \cdot 2$ & $304 \cdot 333$ & $0 \cdot 442364$ & $-492 \cdot 867$ \\
\hline$-0 \cdot 2$ & $0 \cdot 2$ & $327 \cdot 975$ & $0 \cdot 365111$ & $-433 \cdot 94$ \\
\hline$-0 \cdot 2$ & $0 \cdot 1$ & 325.959 & $0 \cdot 374013$ & $-458 \cdot 815$ \\
\hline$-0 \cdot 2$ & $0 \cdot 0$ & $324 \cdot 083$ & $0 \cdot 382171$ & $-489 \cdot 58$ \\
\hline$-0 \cdot 2$ & $-0 \cdot 1$ & $322 \cdot 339$ & $0 \cdot 389647$ & $-526 \cdot 321$ \\
\hline$-0 \cdot 2$ & $-0 \cdot 2$ & $320 \cdot 729$ & $0 \cdot 396525$ & $-569 \cdot 23$ \\
\hline
\end{tabular}

well. Hence the estimated energies can serve as very good starting values for CE-CVM optimization programs.

As in the case of $b c c$ disordered structure, consolute point data have been obtained for $f c c$ disordered structure in the tetrahedron-octahedron approximation by using modified Newton-Raphson method for an initial choice of $e_{1}=-250 \mathrm{~J} \mathrm{~mol}^{-1}$ and $e_{j}=0(j=2$ to 9$)$. These are $T_{\mathrm{c}}$ $=300.903 \mathrm{~K}, x_{\mathrm{c}}=0.5$ and $T_{x x}=-362.215 \mathrm{~K}$. In general, the energies corresponding to all the submotifs containing second neighbour distances have been neglected in the literature. Accordingly, we set $e_{2}=e_{4}=e_{6}=e_{7}=e_{8}=$ $e_{9}=0$ and vary the ratios, $e_{3} / e_{1}$ and $e_{5} / e_{1}$, in the range from $-0 \cdot 2$ to $0 \cdot 2$ in steps of $0 \cdot 1$, and obtain the corresponding consolute point data which are given in table 3 .
Once again by fitting suitable polynomial functions of $e_{3} / e_{1}$ and $e_{5} / e_{1}$ to the tabulated data for $T_{\mathrm{c}}, x_{\mathrm{c}}$ and $T_{x x}$, the following relations have been obtained for $f c c$ structure.

$$
\begin{aligned}
x_{c}^{\mathrm{obs}}= & 0 \cdot 5+0 \cdot 299259\left(e_{3} / e_{1}\right)^{3 / 5}+0 \cdot 212183\left(e_{3} / e_{1}\right)^{3 / 5}\left(e_{5} / e_{1}\right), \\
T_{c}^{\mathrm{obs}} & =e_{1}\left[-1 \cdot 20361-2 \cdot 34275\left(e_{3} / e_{1}\right)^{2}-0.0526217\left(e_{5} / e_{1}\right)\right. \\
& \left.-0 \cdot 501314\left(e_{3} / e_{1}\right)^{2}\left(e_{5} / e_{1}\right)\right], \\
T_{x x}^{\mathrm{obs}} & =e_{1}\left[1 \cdot 44886+13 \cdot 531\left(e_{3} / e_{1}\right)^{2}-1.79837\left(e_{5} / e_{1}\right)\right. \\
& \left.+11.2606\left(e_{3} / e_{1}\right)^{2}\left(e_{5} / e_{1}\right)\right] .
\end{aligned}
$$

Once again, this set of equations can be solved in a manner similar to that of the $b c c$ case. Further, this procedure has been verified to be valid and the differences among observed and estimated data have been well within the permissible limits.

The empirical fits given above span the range -0.2 to $+0 \cdot 2$ for the energy parameter ratios and cover an asymmetry of the $x_{\mathrm{c}}$ which is commonly observed in real systems. While the relations found here may be valid for small extrapolations outside the domain considered, this procedure can be extended by considering wider ranges of energy parameter ratios to cover greater degree of asymmetry of the $x_{c}$, depending on a specific requirement. It may be noted that the solution of the (empirical) polynomial equations given above involves NR iterations in itself. Hence care should be exercised to obtain real and meaningful solutions for the energy parameter ratios.

It may be pointed out that the modified NR (presented in $\S 3$ ) for the case of a double root in $x$ and a simple root in $T$ is applicable to the determination of two other special points in phase diagrams: (i) critical point of an order-disorder boundary (first order transition)-This is given by $\Delta G(x, T)=G^{\alpha}-G^{\beta}=0$ and $\Delta G_{x}(x, T)=G^{\alpha}{ }_{x}-$ $G^{\beta}{ }_{x}=0$, where one of the phases is ordered, the other being disordered and (ii) congruent extrema in two phase boundaries. This case is very similar to that of the critical point in (i) except that the two phases involved need not be structurally related to each other. 


\section{Conclusions}

A method has been devised to establish empirical relations among CE-CVM energy parameters and the observed consolute point data from phase diagrams. Using these relations, good initial estimates of parameters can be obtained. These parameters reproduce the observed topology of the phase diagrams and can thus be used for subsequent simultaneous optimization of thermodynamic, structural and phase equilibria data for materials systems. Further, a necessary modification to the classical NR method for solving simultaneous nonlinear/transcendental equations with a double root in one variable and a simple root in the other has been presented. Three applications of the modified NR method to find special points in phase diagrams have also been pointed out.

\section{Acknowledgements}

This investigation forms part of the Ph.D. research of the first author. He is grateful to the Principal and the management of the VNR Vignanajyothi Institute of Engineering and Technology, Kukatpally, Hyderabad, for giving him study leave for pursuing Ph.D. research at the Banaras
Hindu University, Varanasi. He is also grateful to the Head, Department of Metallurgical Engineering, for providing facilities. Financial support through a senior research fellowship from the Institute of Technology is also acknowledged.

\section{References}

Ackermann H, Inden G and Kikuchi R 1989 Acta Metall. 371

Barker J A 1953 Proc. R. Soc. A216 45

de Fontaine D 1994 Solid State Phys. 4733

Inden G and Pitsch W 1991 in Materials science and technology (ed.) P Haasen (Weinheim: VCH, Verlagsgesellschaft) Vol. 5, pp. 497-552

Kikuchi R 1951 Phys. Rev. 81988

Lupis C H P 1983 Chemical thermodynamics of materials (Amsterdam: North Holland) pp. 225-226

Mathews J H 1998 Numerical methods for mathematics, science and engineering (New Delhi: Prentice-Hall) 2nd ed.

Sanchez J M and de Fontaine D 1978 Phys. Rev. B17 2926

Sanchez J M, Ducastelle F and Gratias D 1984 Physica $\mathbf{A 1 2 8}$ 334

Saunders N and Miodownik A P 1998 CALPHAD (Calculation of phase diagrams): A comprehensive guide (Oxford: Pergamon) 